\title{
POLYMOLDING: TWO WAFER POLYSILICON MICROMOLDING OF CLOSED- FLOW PASSAGES FOR MICRONEEDLES AND MICROFLUIDIC DEVICES
}

\author{
Neil H. Talbot and Albert P. Pisano \\ Department of Mechanical Engineering \\ University of California at Berkeley \\ Berkeley, CA 94720
}

\begin{abstract}
A two wafer polysilicon micromolding process we call Polymolding has been developed that permits the fabrication of hollow tubes and other structures useful for fluidic systems and relatively large mechanical structures. A related process known as HexSil, developed by Chris Keller at UC Berkeley, involves the deposition of an oxide release layer and polysilicon on a single mold wafer from which polysilicon structures are subsequently released [1]. In contrast, this new process uses two mold wafers that are temporarily bonded together after the deposition of the oxide release layer. The two wafers form a complete mold with internal chambers. Then amorphous polysilicon is vapor deposited and coats the internal chambers of the mold via access holes in the top mold wafer. After some further processing, the wafers are separated and the structures are released. This process is particularly useful for fluidic applications because complex closed flow passages, such as microfilters, interconnected chambers, fluid manifolds etc. can be easily be created. Polysilicon microneedles from 100 to 200 microns in diameter and $1 \mathrm{~mm}$ to $7 \mathrm{~mm}$ in length and sub-micron tip radii have been fabricated using this process. These microneedles are valuable for small volume drug injection where minimal tissue damage and/or minimal pain are desired. In addition, the Polymolding process does not damage the mold wafers, so they can be reused many times at a huge cost savings.
\end{abstract}

\section{INTRODUCTION}

The primary motivation for the development of Polymolding and Polymolded microneedles is the need for very small hypodermic injection needles that are economical to fabricate. Small needles are very desirable because they reduce insertion pain and tissue damage in the patient. This is particularly important for Type I diabetics who typically have to inject themselves with insulin 3 times a day. Currently the smallest needles available for diabetics are 30 gauge needles which are 305 microns in diameter with a wall thickness of 76 microns. Traditional machining techniques are unable to produce a viable needle with a significantly smaller diameter than 300 microns. The typical needle material, medical grade stainless steel, is simply too ductile when the wall thickness is only tens of microns. In addition, there are various designs of small needles that are impossible or impractical to fabricate with traditional machining techniques. For example sharp, $90^{\circ}$ bends are impossible to machine out of drawn steel tubes because they always crimp at the bend and close the fluid flow passage. Needles with multiple fluid channels are also very difficult to machine. However, with silicon micromachining, microneedles with these, and other useful features are easily fabricated. Extreme sharpness can also be achieved with Polymolded microneedles (Fig. 1) while stainless steel needles can be comparatively rough (Fig. 2).

The early research on microfabricated microneedles was done by Liwei Lin at the University of California at Berkeley [2] and
Kensall Wise at the University of Michigan [3]. In both of these approaches the microneedles were made of single crystal silicon. The device wafer was sacrificed or dissolved away in a silicon etchant leaving the microneedles behind. Wise' approach took advantage of the slow etch rate of heavily boron doped silicon in EDP. Lin's approached used both the differential etch rate of EDP and a timed etch to produce the desired needle shape. The main contrast and key advantage of Polymolding in that the mold wafers are not sacrificed during the fabrication process. Since the process is a molding process, the mold wafers can be re-used many times at a huge cost savings as compared to sacrificial processing techniques.

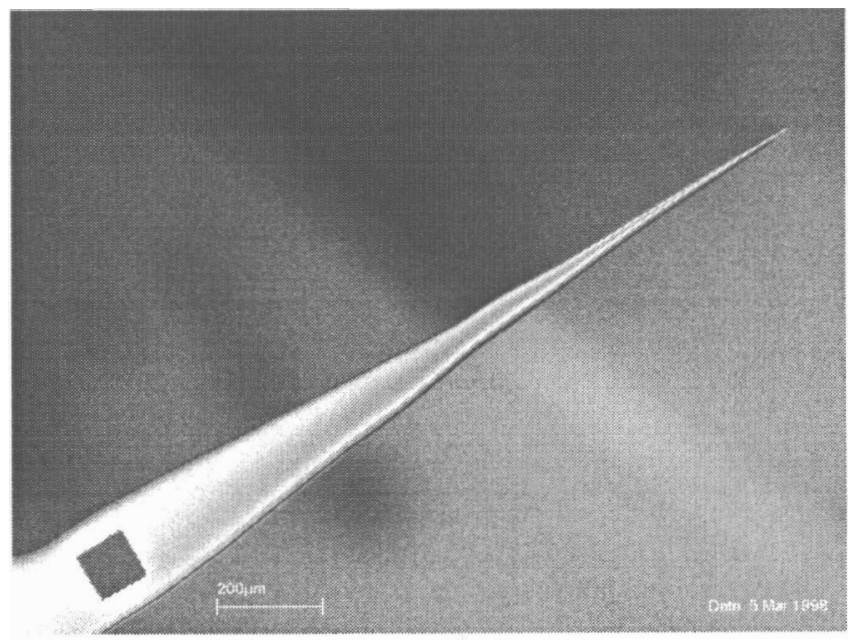

Figure 1. SEM of the tip of a Polymolded microneedle with sub. micron tip radii.

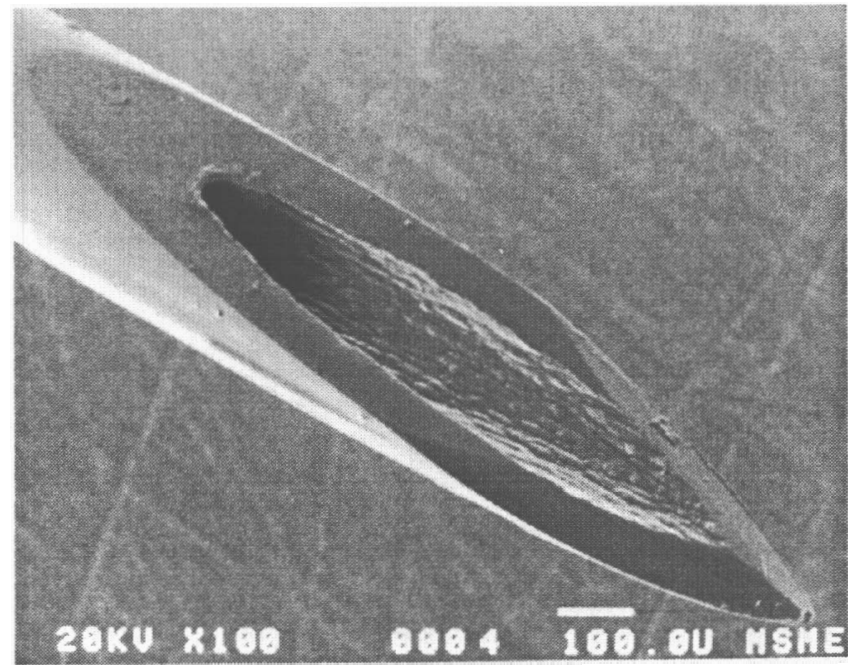

Figure 2. SEM of the tip of a 30 gauge stainless steel needle. 


\section{FABRICATION}

Polymolding is a two wafer molding process (Fig. 3). Polysilicon is used as the structural material for the parts, which in this case were microneedles. The molds were made from lightly doped, p-type, (100), single-crystal silicon wafers. Up to this point $100 \mathrm{~mm}$ wafers have been used, but this process is easily scalable to larger wafers. Double-sided polished wafers were used for the top mold wafers. The top mold wafer was coated with 0.3 microns of LPCVD low-stress silicon nitride that was then patterned with a dark field mask to expose through-hole regions. After the nitride was etched away in these regions, the photoresist was stripped and the through-holes were etched with $\mathrm{KOH}$. The remaining silicon nitride was removed with concentrated HF.

The bottom mold wafers were masked with about 2 microns of photoresist and then patterned. They were then deep-trench etched in a Surface Technology Systems (STS) silicon etcher to define the microneedle body shapes and features. Typical etch depths were 100 to 130 microns. The etch recipe required careful tuning for each die design to obtain a desirable etch profile. Unoptimized recipes produced re-entrant (undercut) etch profiles which required very thick release layers to ensure that the microneedles could be removed from the mold. A thick release layer means that the minimum feature size ends up being much larger than normal, which was naturally undesirable for intricate designs. Typically both top and bottom mold wafers were coated with 2 microns of PSG (Fig. 4B). After the PSG was densified, bottom mold wafers requiring a thicker release layer were coated with 0.5 microns of $580^{\circ} \mathrm{C}$ polysilicon which was then oxidized to form 1 micron of oxide. Then wafers were aligned and pressure bonded in nitrogen at $1000^{\circ} \mathrm{C}$ (Fig. 4C).
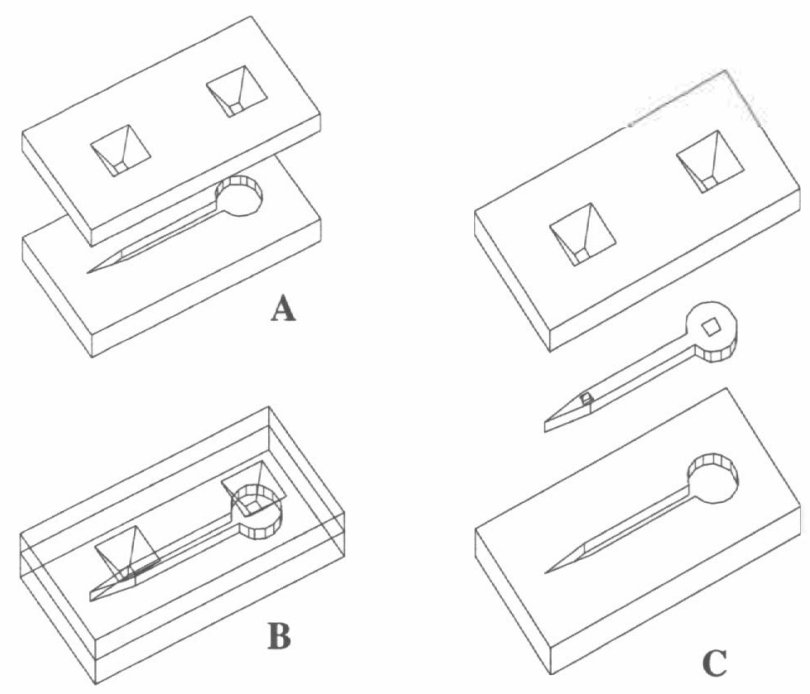

Figure 3. Oblique view of the mold wafers prior to bonding (A), after bonding $(B)$, and after the final release step $(C)$.

After bonding, 3 microns of LPCVD polysilicon were deposited on the mold wafers at $580^{\circ} \mathrm{C}$ (Fig. 6A). This low deposition temperature was used in order to produce amorphous polysilicon that has desirable mechanical properties when annealed and deposits extremely conformally. The molds were then annealed in nitrogen for 1 hour at $1000^{\circ} \mathrm{C}$. Then the next layer of polysilicon was deposited and annealed. These deposition and annealing steps were repeated until the desired thickness of polysilicon was achieved -typically 12 to 18 microns.

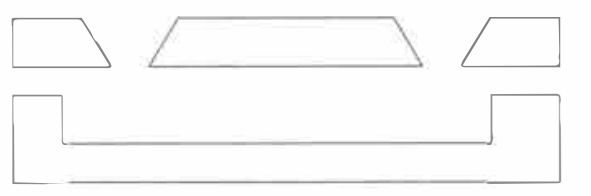

A

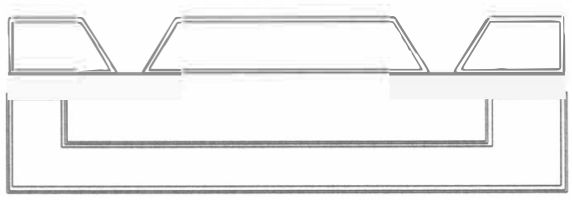

B

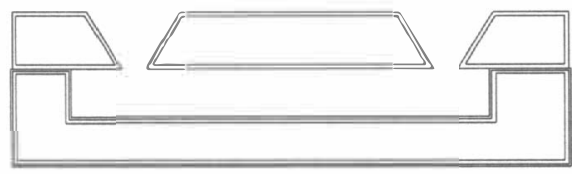

C

Figure 4. Mold wafers as fabricated (A); after application of the release layer $(B)$; after bonding $(C)$.

The next step was to anisotropically remove the polysilicon on the top of the mold using the STS etcher. A Lam polysilicon etcher has also been used for this step. The surface polysilicon was etched until it cleared and then over-etched by $25 \%$ to $100 \%$. Some batches were over-etched only $25 \%$, so there was still a thin polysilicon "funnel" structurally attaching the microneedles to the top mold wafer. After the final release etch these microneedles remained loosely attached to the top mold wafer for greater ease of handling and/or post processing (Fig. 5).

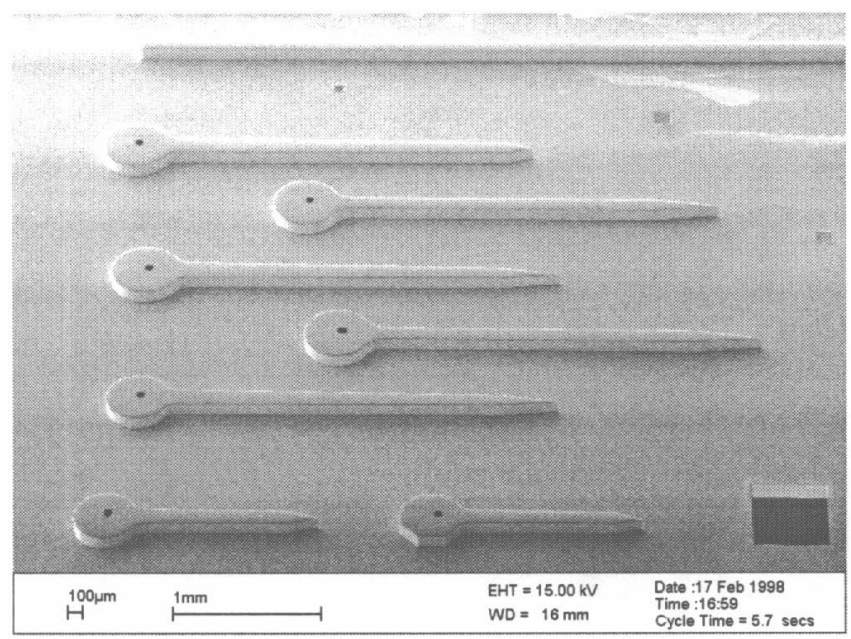

Figure 5. SEM of a group of released microneedles loosely attached to the top mold wafer for ease of handling

During the final release etch in concentrated HF, the wafers were separated and the microneedles were liberated from the mold with a release etch. Release times varied from a few hours to overnight depending on the mold geometry and the separation of the etch holes. 


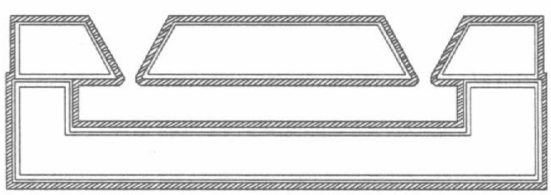

$\mathbf{A}$

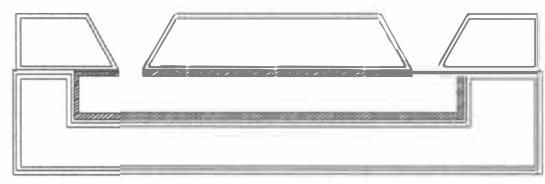

B

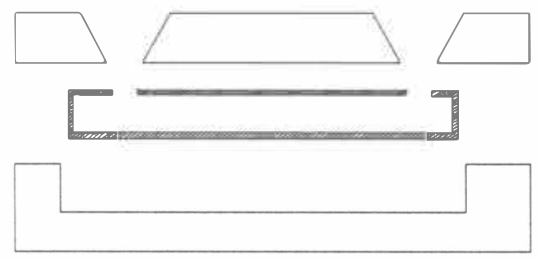

C

Figure 6. Mold wafers after polysilicon deposition (A); after external polysilicon removed (B); after the release step $(C)$.

\section{RESULTS AND DISCUSSION}

Four batches of microneedles have been fabricated with minor variations in the described process flow. The first two batches had release layers for the bottom mold wafer that were composed of 2 microns of PSG and two subsequent layers of oxidized polysilicon each 1 micron thick. The top mold wafer simply had 2 microns of PSG. The microneedle designs of these first two batches were fairly simple due to the thickness of the release layer. The most intricate design was a dual channel microneedle. The thick release layer was necessary because of the poor quality of the out-sourced deep-trench etching of the bottom mold wafer. The first batch had a polysilicon wall thickness of 12 microns (Fig. 7) and their cross-section was 100 microns square with a slight taper. These microneedles were too weak to be handled easily. The second batch had a polysilicon wall thickness of 18 microns (Fig. 5 and was much stronger, being able withstand bending moments of up to $0.53 \mathrm{mNm}$. Some of these microneedles were also electroplated or sputtered with metals such as nickel (Fig. 7) and could withstand bending moments of up to $0.71 \mathrm{mNm}$.
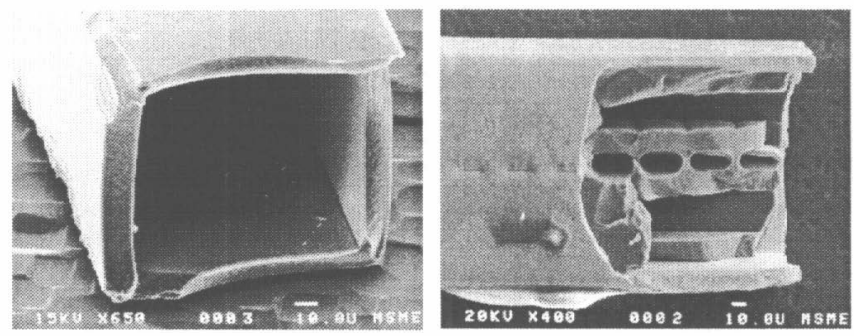

Figure 7. SEM of the cross-section of a microneedle from batch one (left). SEM of the internal structure of a metal plated dual channel microneedle (right).

In order to make the microneedles stronger the molds for batches 3 and 4 were redesigned. The principal design changes were that the diameter of the microneedles was increase to 200 microns in the plane of the wafer and 110 microns out of plane and also internal wall reinforcement features were added (Fig. 8).
These detailed features demonstrate the remarkable flexibility of this process.

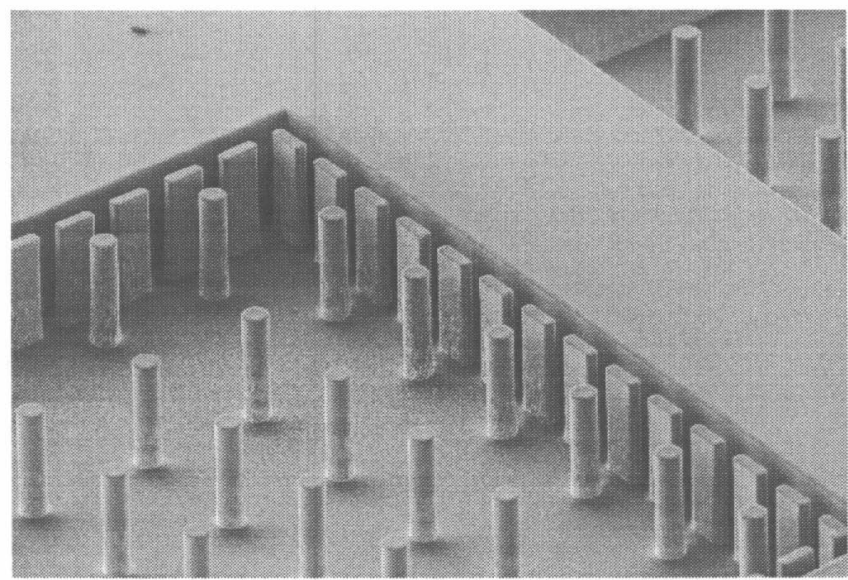

Figure 8. SEM of a mold showing strength enhancement features.

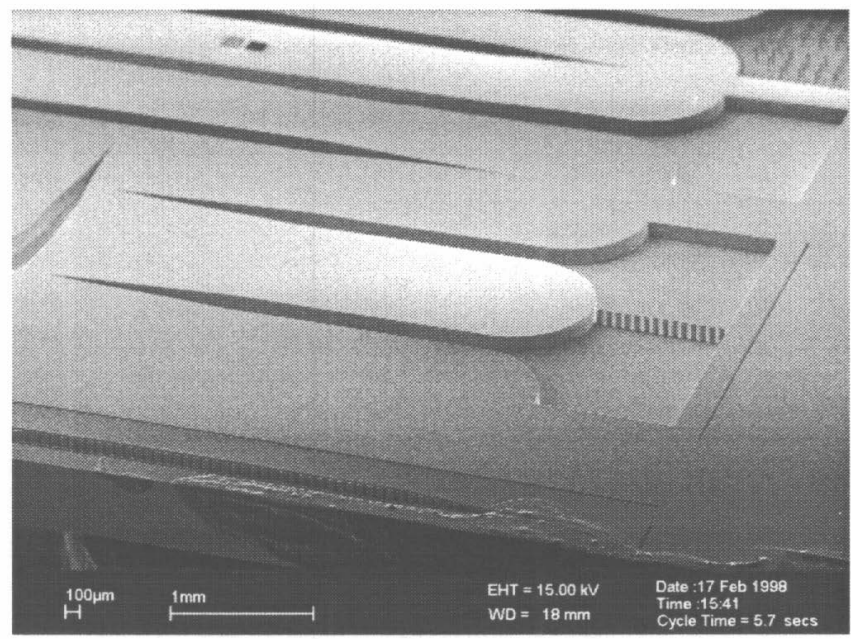

Figure 9. SEM of a dual shaft microneedle mold.

The third batch of microneedles had a release layer of 3 microns of PSG. After the bonding step, 0.3 micron of polysilicon was deposited and then oxidized in order to reduce or eliminate the thin layer of polysilicon that tends to form where the halves of the mold meet. This thin layer of extraneous polysilicon, known as mold flash, was not significantly reduced by the oxidized polysilicon, so other solutions are being investigated. These microneedles were not very strong because of separation of the layers of polysilicon in the needles. Due to improvements in the deep-trench etching recipe the release layer for the fourth batch was reduced to only 1.5 microns of PSG and 0.6 microns of oxidized polysilicon. Several unique microneedle designs were included in the last two batches such as a dual shaft microneedle (Fig. 9, Fig. 10) and a microneedle with filter in the base (Fig. 11, Fig. 12). 


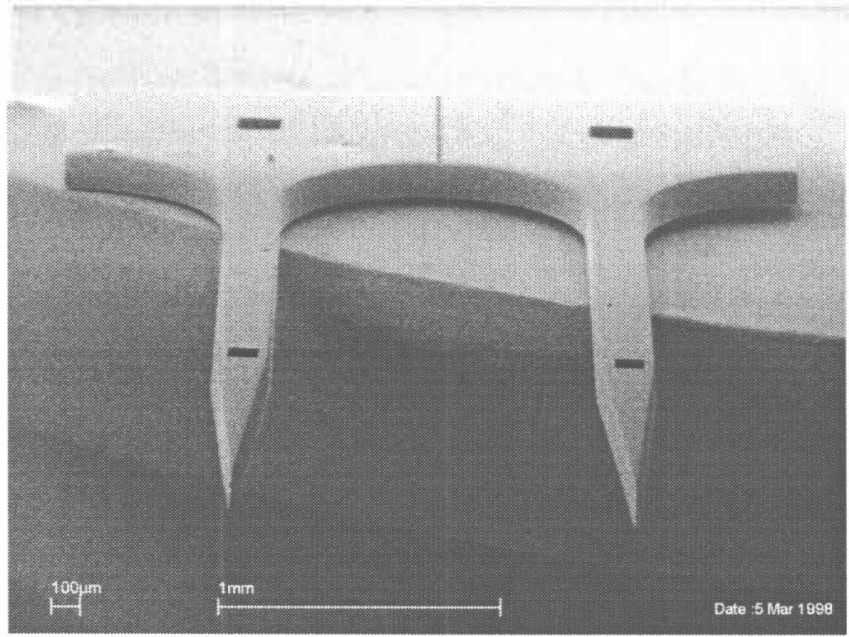

Figure 10. SEM of a $4 \mathrm{~mm}$ long dual shaft microneedle.

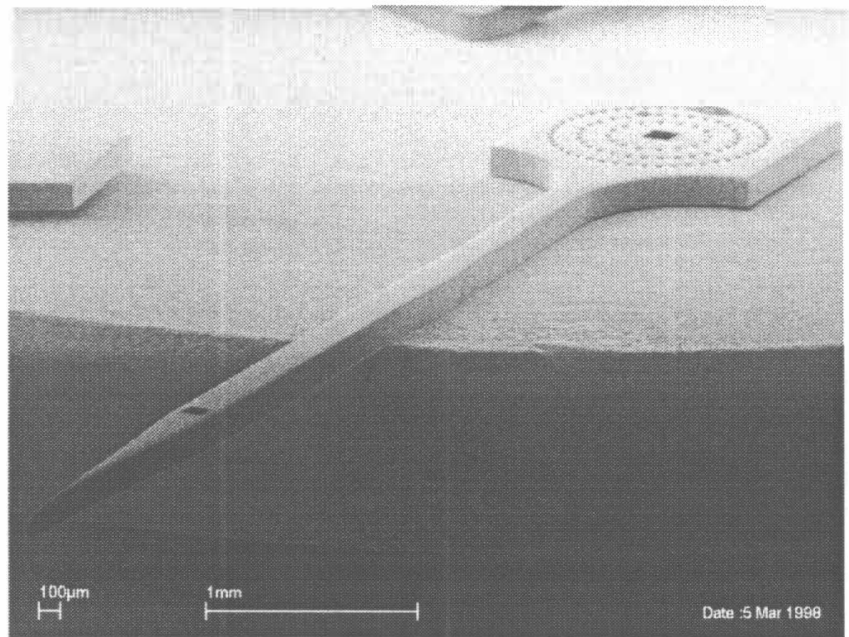

Figure 11. SEM of a microneedle with a $6 \mathrm{~mm}$ shaft and a microfilter in its base.

\section{CONCLUSIONS}

Polymolding, a versatile process for the fabrication of microneedles and other microfluidic and mechanical devices has been developed and demonstrated. Polysilicon microneedles 100 to 200 microns in outer diameter have been fabricated that are capable of supporting bending moments of up to $0.71 \mathrm{mNm}$. This new pro-

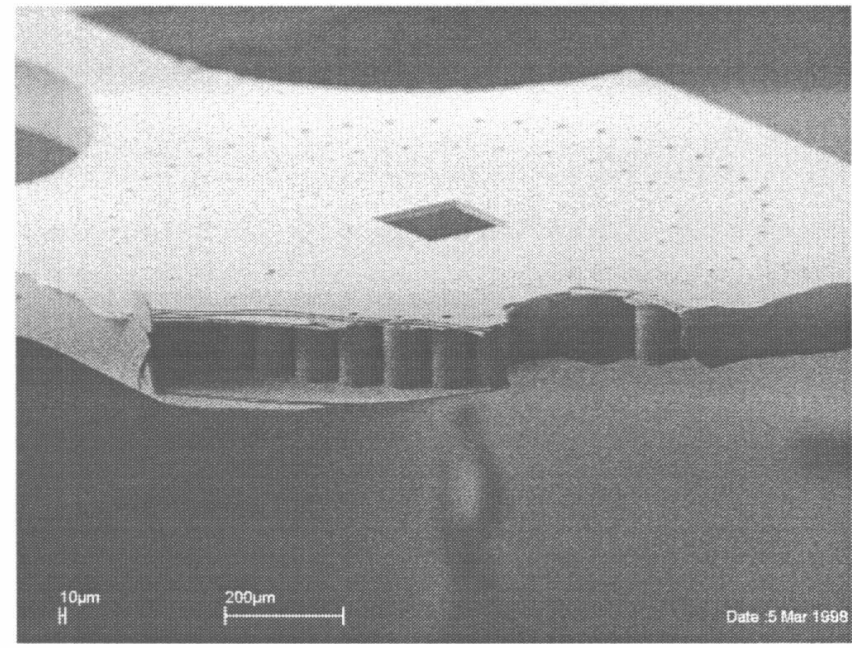

Figure 12. Close-up of the base of microneedle with a builtin filter that has been cleaved.

cess is extremely flexible especially when compared to traditional machining techniques because detailed structures can be fabricated such as microfilters, interconnected chambers, fluid manifolds etc. In addition, for the fabrication of microneedles, this process is more economical than other microfabrication processes because the device wafer (or mold) is re-usable.

\section{ACKNOWLEGEMENTS}

This research was supported by the Becton Dickinson Research Center. Contact: Burton Sage

\section{REFERENCES}

1. C.G. Keller, R.T. Howe, "Nickel-filled hexsil thermally actuated tweezer," International Solid-State Sensors and Actuators Conference, Transducers ‘95, Stockholm, Sweden, pp.376-379

2. L. Lin, A.P. Pisano, R.S Muller, "Silicon processed microneedles," International Solid-State Sensors and Actuators Conference, Transducers ‘93, Japan, pp. 237-240

3. J. Chen, K.D. Wise, “A multichannel neural probe for selective chemical delivery at the cellular level," Solid State Sensor and Actuator Workshop, Hilton Head, S.C., (1994), pp. 256-259 